\title{
A Importância do Coaching Pessoal e Profissional como Diferencial Competitivo para o Mercado de Trabalho
}

\section{The Importance of Personal and Professional Coaching as a Competitive Differential for the Labor Market}

\author{
Eduardo Mathias Cristello; Michele Ventura Cristello*b \\ anstituto Federal de Educação Tecnológica do Rio Grande do Sul. RS, Brasil. \\ ${ }^{\mathrm{b}}$ Faculdade Anhanguera do Rio Grande. RS, Brasil. \\ *E-mail: mi.cristello@hotmail.com
}

\begin{abstract}
Resumo
O Coaching pessoal e profissional tem sido muito utilizado atualmente em função dos benefícios que promove à vida pessoal e profissional dos indivíduos, possibilitando uma maior adequação ao novo perfil profissional exigido pelo mercado, bem como um diferencial competitivo a este profissional. Assim, este trabalho visa apresentar alguns estudos que evidenciam a importância do Coaching pessoal e profissional como diferencial competitivo para mercado de trabalho. Por meio de uma pesquisa bibliográfica, pode-se observar que o Coaching pessoal e profissional mostrou-se como ferramenta capaz de auxiliar os profissionais em seu desenvolvimento, na realização de metas e projetos, no alcance de resultados, promovendo desta forma melhorias no desempenho profissional, o que contribui significativamente para trazer ao profissional um diferencial competitivo no mercado de trabalho.
\end{abstract}

Palavras-chave: Coaching Pessoal e Profissional. Mercado de Trabalho. Desenvolvimento Pessoal e Profissional. Perfil Profissional

\begin{abstract}
The Coaching staff has been very professional and currently used in relation to the benefits that promotes the personal and professional lives of individuals, allowing greater adjustment to the new job profile required by the market, as well as a competitive differentiator in this work. Thus this paper presents some studies that show the importance of personal and professional Coaching as a competitive market for labor. Through a literature search can be seen that the Coaching staff and showed up as a professional tool to assist practitioners in their development, the achievement of goals and projects, in achieving results, thus promoting improvements in work performance, the which contributes significantly to bring the professional in a competitive labor market.
\end{abstract}

Keywords: Personal and Professional Coaching. Job Market. Personal and Professional Development. Professional Profile.

\section{Introdução}

O cenário atual do mercado de trabalho está consideravelmente diferente daquele de algumas décadas passadas. Nota-se que a competitividade, a globalização e as metas trazem novas demandas aos profissionais, tais como capacidade de aprender, criatividade, iniciativa, novas competências e habilidades, melhores relações interpessoais, enfim, um novo perfil profissional.

Segundo Chiavenato (2017), o cenário de mudanças, a globalização da economia, a competitividade e os intensos programas de produtividade e qualidade estão trazendo modificações ao papel dos profissionais nas empresas, alterando de forma significativa o perfil profissional dos funcionários. Aqueles que não se ajustam a esse novo perfil estão fadados à perda do emprego para outro que esteja mais adequado às novas exigências e expectativas do mercado.

O profissional para ingressar, se manter ou ainda se destacar no mercado de trabalho, diante do cenário de mudanças atual, precisa se adequar ao novo perfil profissional exigido, desenvolvendo características essenciais ao mesmo. Corroborando com esta afirmação, Bernhoeft (2002) assinalou que o trabalhador de uma forma geral, alicerçou- se em suas competências e habilidades, fazendo delas sua vantagem competitiva, consolidando cada pessoa como única, bem como diferenciada, de maneira que se torne hábil para responder às mudanças e oscilações do contexto com a velocidade e adequação necessários.

Para Chiavenato (2017) o emprego está se tornando cada vez mais raro, e para se manter nele, as pessoas terão suas funções e atividades modificadas para que dessa forma acompanhem a evolução do mercado. Uma vez que a competitividade vem exigindo dos indivíduos um investimento constante em sua carreira e em sua preparação e qualificação profissional.

Conforme Bernhoeft (2002), apesar do cenário atual aparentar extenuante e insensível, com ele surgem ferramentas de relacionamento e humanização da prática organizacional, que elevam o profissionalismo e a performance geral e, o Coaching é uma delas. O Coaching, para o mesmo autor, "é uma das melhores ferramentas para crescer e fazer crescer. Sua prática é um exercício de inspiração e estímulo, uma humanização constante da relação entre duas pessoas, sobre uma busca" (2002).

Diante do acima exposto justifica-se a relevância deste estudo sobre Coaching, pois esta metodologia tem ganhado 
espaço no meio acadêmico, empresarial e cada vez mais adeptos a seus conceitos.

Por meio deste estudo buscou-se responder ao seguinte questionamento: De que maneira os indivíduos poderão desenvolver o perfil profissional exigido por um mercado de trabalho cada vez mais competitivo?

Para tanto esta pesquisa teve como objetivo geral compreender de que forma o Coaching pode ser usado como ferramenta que viabilize aos indivíduos um diferencial competitivo no mercado de trabalho, e como objetivos específicos, identificar os tipos de Coaching, conceituar o Coaching pessoal e profissional e descrever as contribuições do Coaching para o desenvolvimento pessoal e profissional dos indivíduos.

Em função dos objetivos deste estudo, tornou-se mais indicada a escolha da pesquisa bibliográfica, baseada em fontes secundárias retiradas de artigos da internet e livros de autores renomados da área de gestão de pessoas.

\section{Desenvolvimento}

\subsubsection{Metodologia}

Para o desenvolvimento do presente artigo, utilizou-se a pesquisa bibliográfica, por ser a mais indicada ao que se pretende demonstrar.

Para Fachin (2003) a pesquisa bibliográfica diz respeito ao conjunto de conhecimentos humanos reunidos nas obras. Tem como finalidade fundamental conduzir o leitor a determinado assunto e proporcionar a produção, coleção, armazenamento, reprodução, utilização e comunicação das informações coletadas para o desempenho da pesquisa.

Para a seleção dos artigos foi utilizado a base de dados do Google Acadêmico, onde foram utilizadas as palavras chaves "Coaching pessoal"; "Coaching profissional" e a "Importância do Coaching pessoal e profissional" sendo os artigos selecionados por meio da similaridades de ocorrências durante a pesquisa, ou seja ao digitar a palavra chave, foram selecionados os artigos que apareciam em ambas as buscas na base de dados.

Outros artigos, bem como trabalhos de conclusão de curso de graduação e pós-graduação também foram considerados para este estudo além de livros na área de psicologia, gestão de pessoas e áreas afins, que colaboram com o tema.

\subsection{Discussão}

\subsubsection{Coaching: origem, conceito e evolução}

O Coaching embora pareça algo novo não é. Ele já foi utilizado como processo pedagógico de ensinar as pessoas a pensar e refletir em busca de conhecimento no fundo de si mesmo, Sócrates foi seu criador (CHIAVENATO, 2017).

Etimologicamente o Coaching originou-se no mundo dos esportes e assinala o papel de professor, técnico, treinador, preparador. Papel que se assume ao se comprometer em apoiar alguém a atingir algum resultado (ARAÚJO, 2011).
Já o termo Coach, segundo Ricci (2006) teve sua origem no vilarejo de Kocz na Hungria, local esse onde teria sido criada a primeira carruagem com o objetivo de transportar passageiros. Dessa forma, estabelecendo uma analogia, o Coach pode ser considerado alguém que conduz pessoas de um local a outro, isto é, de um estado ou condição atual para um estado ou condição futuro. Enquanto o Coachee trata-se do indivíduo que passa pelo processo de Coaching, o cliente (PEIXOTO, 2012).

Já no mundo empresarial a palavra Coaching segundo Chiavenato (2017) é uma ferramenta, um instrumento gerencial que se utiliza de técnicas não diretivas para estimular o indivíduo a refletir sobre seu comportamento ou decisões, levando a escolher a melhor alternativa para uma determinada situação de trabalho. Visa orientar e otimizar o desenvolvimento da carreira executiva e o aperfeiçoamento pessoal.

O Coaching vem ganhando espaço como uma metodologia que auxilia os indivíduos a se adaptarem ao novo perfil profissional exigido pelo mercado. Conforme Milaré (2004) dentre os diversos motivos que justificam o uso da metodologia de Coaching, o principal deles é sua contribuição para aquisição e o amadurecimento de competências, bem como adaptação aos processos de transformação organizacional com melhoria de desempenho.

As importantes transformações pelas quais as organizações vêm passando, forçadas pela necessidade de competir e sobreviver num mercado dinâmico, globalizado e exigente, deflagram processos de mudança dos indivíduos em nível comportamental (NERI, 2000 apud ELISA; YOSHIDA, 2007). Assim, torna-se imprescindível uma capacitação profissional adequada às novas e crescentes demandas de mercado.

A base metodológica do Coaching, conforme Kunzler e Schneider (2012) pode ser resumida da seguinte forma:

a) Atender o cliente em suas necessidades, explicando o processo de Coaching e estabelecendo um vínculo com o mesmo;

b) Administrar expectativas;

c) Avaliar o cliente, obtendo informações sobre ele;

d) Descobrir a preocupação do cliente;

e) Esboçar a aliança e comprometimento de trabalho;

f) Objetivar o modo de trabalho, como sessões, tempo, prazo, etc.

g) Estabelecer metas, estado desejado e sonho a realizar;

h) Elaborar plano de ação, com metas e objetivos claros;

i) Elencar tarefas a serem realizadas durante as sessões até o alcance de metas e objetivos desejados;

j) Monitorar, avaliar, corrigir e realizar a passagem entre o estado atual, referente ao início do Coaching e o estado desejado, realizando dessa forma o término do processo de Coaching.

Logo, o propósito do Coaching de desenvolver pessoas vem ao encontro das altas exigências feitas aos profissionais da atualidade, promovendo melhores práticas no trabalho e melhorias na vida pessoal. 


\subsubsection{Coaching no cenário atual de mudanças}

A partir da década de 80 as empresas passaram a compreender a importância essencial das pessoas em seus processos. Atualmente há também uma visão de que mais importante do que competência técnica são os aspectos comportamentais, assim como a qualidade das decisões dos colaboradores, que emperravam ou alavancavam o andamento de um negócio. Desta maneira, as organizações passaram a esperar mais de seus profissionais. (ZAHAROV, 2010).

Nesse cenário, ainda segundo o mesmo autor, o treinamento para desenvolver competências interpessoais tornou-se fundamental para melhor preparar o fator humano. Os indivíduos também foram se dando conta de que se quisessem progredir na carreira precisariam "correr atrás" de seu crescimento. Era preciso algo que revolucionasse o aprendizado nas corporações como resposta a todas essas mudanças, e o Coaching trouxe essa possibilidade de as pessoas usarem em uma escala maior seus recursos pessoais, sua capacidade de criar, resolver problemas e produzir resultados com maior rapidez (ZAHAROV, 2010).

Para atingir sucesso profissional é necessário muito mais do que conhecimento específico na sua área de atuação, espera-se também do profissional habilidades múltiplas no que se refere a relações humanas (PAULA, 2005).

Dentro desse cenário, Castel (2013) menciona que ao indivíduo não basta mais saber trabalhar, é preciso saber, tanto quanto vender e se vender. Assim os indivíduos são levados a definir, eles próprios, sua identidade profissional e fazer com que seja reconhecida numa interação que mobiliza tanto um capital pessoal quanto uma competência técnica geral.

Nota-se claramente o quanto é ampla a gama de competências e habilidades esperadas de um profissional atualmente, bem como a premência do desenvolvimento de potencial e talento de cada indivíduo. Logo, mais do que nunca se explica a necessidade de ferramentas de desenvolvimento de pessoas, como o Coaching.

Segundo Chiavenato (2017) o Coaching é um relacionamento em que o Coach se compromete em apoiar e ajudar o aprendiz para que este possa atingir um resultado ou seguir determinado caminho. No entanto, ainda para o autor, o Coaching não se limita a alcance de resultados, mas um compromisso com a pessoa em si, com seu desenvolvimento profissional e sua realização pessoal. O Coaching é um relacionamento que produz novas competências.

O Coaching além de viabilizar uma melhor colocação dentro do mercado de trabalho também contribui para realização pessoal, uma vez que a percepção de sentir-se útil naquilo que faz confere ao ser humano sentido de sua existência. Conforme Bernhoeft (2002), a necessidade vital de sentir-se produtivo, útil, com uma percepção clara e concreta do valor de si mesmo é uma das questões trabalhadas com a ajuda da ferramenta do Coaching. Esse processo de saber realizar, de concretizar, tem um profundo sentido existencial.
Com o processo de Coaching é possível desenvolver e até mesmo superar as principais qualidades e habilidades de um indivíduo. Tal processo utiliza-se de técnicas, ferramentas e recursos de diferentes ciências. O Coaching é um cocktail, um mix de recursos e técnicas que funcionam em ciências do comportamento (psicologia, sociologia, neurociências) e de ferramentas da administração de empresas, esportes, gestão de recursos humanos, planejamento estratégico e outros (PEIXOTO, 2012).

O trabalho do Coach consiste em incentivar as pessoas a obterem melhores resultados no desenvolvimento de competências profissionais e pessoais, por meio de análises individuais, dinâmicas de grupo, atividades para desenvolver a auto-motivação, autodesenvolvimento e autoliderança, possibilitando assim o crescimento (PEIXOTO, 2012).

No relacionamento com o Coach, o Coachee recebe liderança, aconselhamento, treinamento, estímulo, impulso e etc., ele aproveita então o impulso e a direção para aumentar seus conhecimentos, melhorar aquilo que sabe, aprender coisas novas e deslanchar seu desempenho (GASPAR; PORTÁSIO, 2009).

Logo, o desenvolvimento de pessoas é um desafio pessoal e organizacional nos dias de hoje. Os colaboradores vieram a se tornar o grande diferencial competitivo nas organizações. Investir no desenvolvimento de pessoas tornouse uma premissa para o crescimento, tanto pessoal quanto organizacional.

\subsubsection{Tipos de Coaching}

Existem vários tipos de Coaching, mas segundo Dolan, (2015), os mais divulgados atualmente são:

a) Coaching de Negócios: focado no desempenho da organização.

b) Coaching Executivo: Trata-se de uma relação "one to one" entre o Coach e o Coachee, consoante os objetivos intrapessoais deste último na organização em que se insere.

c) Coaching de vida: orientado para o futuro. Auxilia o Coachee a estabelecer e alcançar suas metas pessoais

Segundo Ensminger et al. (2015) o Coaching ainda pode aparecer nas seguintes tipologias:

a) Coaching de conhecimento $\mathrm{O}$ objetivo é fornecer ao Coachee, as competências e conhecimentos necessários a uma área específica.

b) Coaching de competências Permite ao Coachee adotar novas formas de agir, com o fim de aumentar os seus resultados.

c) Coaching pessoal Tem o intuito de auxiliar o Coachee nos seus desafios pessoais, particularmente, sentimentos, atitudes, perceções e autoeficácia.

d) Coaching de resultados Concentra-se em ajudar o Coachee a atingir metas específicas.

e) Coaching de desenvolvimento Foca-se na carreira do Coachee, na aquisição de mais responsabilidades e tarefas. 


\subsubsection{Coaching pessoal e profissional}

O Coaching pessoal ou de vida tem como foco as metas pessoais, pensamentos, sentimentos e ações, e o modo como um indivíduo poderá mudar sua vida, para que se obtenha uma maior satisfação pessoal (ELISA; YOSHIDA, 2007).

Ainda neste sentido, a meta do Coach pessoal é ajudar o Coachee a ser mais, extrair seu potencial máximo, orientálo para lidar melhor com o futuro e torná-lo um funcionário mais valioso. O Coach também auxilia na mudança de comportamentos que possam ameaçar ou descarrilar sua carreira (BENTON, 2000).

Segundo Peixoto (2012), o Coaching pessoal visa a capacitação das pessoas em sua auto-realização, a meta a ser alcançada pode ser em qualquer área da vida de um indivíduo, como saúde, relacionamentos, espiritualidade, finanças, carreira, administração do tempo, família, etc. Existem vários nichos de Coaching pessoal atualmente, dentre eles: Coaching de casamento ou de noivas, para emagrecimento, para jovens, financeiro e afins.

Conforme Gaspar e Portásio (2009) o Coaching de vida hoje em dia representa tendência mundial em desenvolvimento humano para se alcançar sucesso pessoal e profissional".

Enquanto que o Coaching Profissional, segundo a Federação Internacional de Coaching (ICF- International Coach Federation) apud Oliveira (2007), é um relacionamento contínuo, de alto nível que auxilia as pessoas a obterem resultados extraordinários em suas carreiras, vidas, organizações ou negócios. Por meio dele, os clientes aprofundam seus conhecimentos, aumentam sua performance e aprimoram sua qualidade de vida.

Cabe dizer que tanto o Coaching de vida quanto o profissional ao lançar foco sobre um problema, situação ou desejo específico, estabelecer um plano de ação e uma mudança de comportamento, possibilita a passagem de um estado atual para um estado desejado, ou seja, promove uma mudança, o que contribui consideravelmente para a promoção da qualidade de vida pessoal e para o crescimento e realização profissional.

\subsubsection{Coaching: contribuições para o desenvolvimento pessoal e profissional do indivíduo}

Uma pesquisa realizada nos E.U.A em 2001 junto à "Fortune 500", listou as empresas melhor sucedidas dos E.U.A que utilizaram Coaching, mostrou alguns dados e resultados: incremento em $53 \%$ na produtividade, melhora no atendimento ao cliente em $39 \%$, redução dos custos em $23 \%$, além de melhorar o trabalho de equipes, o gerenciamento de líderes, o relacionamento com clientes e a redução de conflitos (OLIVEIRA, 2007).

No que se refere aos benefícios do Coaching, alguns autores ainda relatam melhoras em autocontrole emocional, aferição de resultados organizacionais, mudança comportamental e desenvolvimento de competências de liderança (TOBIAS,
1996; MILARE; YOSHIDA, 2009 apud CAMPOS; PINTO, 2012).

Conforme Lobo (2011) o Coaching de vida pode trazer benefícios tais como aumento do nível de conquistas, felicidade e plenitude, melhoria nos relacionamentos e na comunicação, melhoria nos resultados financeiros, aumento da autopercepção e do autoconhecimento, melhoria nos focos, planejamento e administração do tempo, aumento da percepção, criatividade e intuição.

E o Coaching profissional pode promover melhoria no nível de satisfação com o trabalho, melhoria no trabalho em equipe, melhoria nas habilidades interpessoais, comportamentais e crenças, melhoria na liderança, aumento do autoconhecimento e da percepção dos outros, desenvolvimento da melhoria de competências e habilidades como liderança, planejamento, pensamento estratégico, comunicação, resolução de problemas e conflitos, bem como o desenvolvimento ou estímulo de qualidades ligadas ao sucesso, tais como capacidade cognitiva, capacidades sociais, estilos de personalidade, motivação e conhecimento (LOBO, 2011).

\section{Conclusão}

Este estudo conceituou a ferramenta Coaching, verificou a sua evolução enquanto metodologia para desenvolver pessoas dentro e fora das organizações, apresentar os tipos de Coaching existentes tais como: o Coaching executivo, Coaching de negócios, Coaching de vida, Coaching de carreira, etc. E por fim além de conceituar os tipos de Coaching pessoal e profissional, buscou-se demonstrar também as principais contribuições dos mesmos tanto para as pessoas, quanto para as organizações.

O Coaching mostrou-se como uma ferramenta capaz de auxiliar os profissionais a buscarem se adequar ao novo perfil de profissional que se deseja. Pois sua metodologia possibilita mudanças tanto pessoal quanto profissional, que viabiliza o alcance de metas e a realização de projetos, o que por vezes torna-se inalcançável sem o uso de ferramentas adequadas.

Além de contribuir para adequação ao novo perfil do mercado o Coaching também auxilia no alcance de resultados, quando foca em dificuldades específicas e possibilita um aprimoramento de características que vêm a emperrar o desempenho profissional.

Atualmente o desenvolvimento de competências e habilidades, tais como liderança, criatividade, capacidade de aprender, solução de conflitos, etc., é fundamental para o crescimento profissional e o uso do Coaching mostrouse como uma ferramenta que foca no trabalho com as mais variadas competências e habilidades. Um dos focos de trabalho do Coaching são as relações interpessoais, trabalho este que desenvolve o indivíduo em competências comportamentais que muitas vezes impedem o crescimento pessoal e profissional, apesar de os mesmos serem excelentes em competências técnicas.

Diante do exposto percebeu-se que o Coaching apresenta- 
se como uma ferramenta de desenvolvimento humano capaz de ir ao encontro de uma demanda de mudança de perfil profissional e desta forma capacitar os indivíduos para não só manterem-se, mas também destacarem-se em um mercado de trabalho cada vez mais competitivo.

\section{Referências}

ARAÚJO, A. Coach: um parceiro para o seu sucesso. São Paulo: Gente, 2011

BENTON, D.A. Faça o que eles fazem: técnicas de Coaching para o desenvolvimento profissional. São Paulo: Negócio, 2000.

BERNHOEFT, R.E.A. Ferramentas de Humanização. In: BOOG, G.; BOOG, M. Manual de gestão de pessoas e equipes: estratégias e tendências. São Paulo: Gente, 2002. p.77-91.

CAMPOS, T.M.; PINTO, H.M.N. Coaching nas organizações: uma revisão bibliográfica. Reuna, v. 17, n. 2, 2012.

CASTEL, R. As metamorfoses da questão social: uma crônica do salário. Petrópolis: Vozes, 2013.

CHIAVEnato, I. Construção de talentos: Coaching \& Mentoring. Rio de Janeiro: Elsevier, 2017.

DOLAN, S. Coaching por valores: um guia para sucesso na vida dos negócios e no negócio da vida. São Paulo: B7, 2015

ELISA, S.A.M.; YOSHIDA, M.P. Coaching de executivos: adaptação e estágio de mudança. Rev. Psicol. Teoria Prática, v.9, n.1, 2007.

ENSMINGER, D.C. et al. Case study of an evaluation Coaching model: exploring the role of the evaluator. Evaluation and Program Planning, v.49, p.124-136, 2015.

FACHIN, O. Fundamentos de metodologia. São Paulo: Saraiva, 2003.
GASPAR, D.J.; PORTÁSIO, R.M. Liderança e Coaching: desenvolvendo pessoas, recriando organizações. Rev. Ciênc. Gerenciais, v.13, n. 18, 2009.

KUNZLER, C.E.B.; SCHNEIDER, E. Coaching: Uma metodologia para qualificação e desenvolvimento profissional. Rev. Destaques Acad., v.4, n.1, 2012.

LOBO, R. Coaching profissional e pessoal. 2011 Disponível em: http:/www.artigopt.com/auto-ajuda/Coaching/Coachingpessoal-e-Coaching-profissional.html Acesso em: 29 mar. 2019.

MILARÉ, S.A. Investimento com retorno garantido. Rev. T\&D Inteligência Corporativa, v.132, n.12, p.20-22, 2004.

OLIVEIRA, E.M. O Coaching apresenta alguma base científica? In: SEMINÁRIO DE GESTÃO DA UNIFAE, 3. 2007. Disponível em: http://cacphp.unioeste.br/eventos/Coaching/ arqs/o_Coaching_BASE_CIENTIFICA.pdf Acesso em: 28 mar. 2019.

PAULA, M. O sucesso é inevitável: Coaching e carreira. São Paulo: Futura, 2005.

PEIXOTO, M.N. Liderança através da ferramenta Coaching: treinando pessoas, desenvolvendo equipes com eficácia. Porto Alegre: Anhanguera, 2012.

RICCI, R. O que é Coaching e como ele pode transformar você? São Paulo: Qualitec, 2006.

SILVA, C.R.E. Orientação Profissional, mentoring, Coaching e counseling: algumas singularidades e similaridades em prática. Rev. Bras. Orientação Prof., v.11, n.2, 2010. Disponível em: http://pepsic.bvsalud.org/pdf/rbop/v11n2/v11n2a14.pdf Acesso em: 28 ago. 2019.

ZAHAROV, A. Coaching: caminhos para a transformação da carreira e vida pessoal. São Paulo: Brasport, 2010. 